\title{
P300 ERP Component on Eating Habits Profiling Using Dynamic Evolving Spiking Neural Network
}

\author{
Cut Amalia Saffiera, Raini Hassan, Amelia Ritahani Ismail \\ Department of Computer Science, Kulliyyah of ICT, International Islamic University Malaysia, Kuala Lumpur, Malaysia \\ amalia.ssafiera@gmail.com, hrai@iium.edu.my, amelia@iium.edu.my
}

\begin{abstract}
Unhealthy eating habits have become a big issue that often causes many chronic diseases in various countries in recent years. The current assessment to identify the status of eating habits is to use selfassessment. However, self-assessment is known to have an error or uncertainty value due to cognitive factors from respondents that affect the results of the assessment. This study identifies a person's eating habits by taking further analysis on the P300 which is an ERP component that excels in showing differences in individual responses to attention processing in visual food images. A set of healthy and unhealthy food images was used as a stimulus when recording the EEG data. The method used for classification is dynamic evolving spiking neural network (deSSN) based on the Neucube architecture. The results showed that the mean amplitude of the P300 component discovered in the Parietal and Occipital lobes was higher for healthy food in the healthy eating habits group. Whereas the unhealthy eating habits group was higher for unhealthy foods. The deSNN classification is proven to operate in learning ERP data but the accuracy rate is not too high due to inadequate sample training.
\end{abstract}

Keywords - P300, Event-Related Potential, Healthy Eating, Dynamic Evolving Spiking Neural Network, DESNN

\section{INTRODUCTION}

Unhealthy eating habits are known widely have a significant effect on health problems [1]. Nevertheless, in the real life there are still many people who are negligent and do not take more attention in maintaining their diet. Fulfill the daily nutritional needs is a very important obligation and requirement to make the body function optimally [2]. This is evidenced by the increasing rates of being overweight and obese over the past few decades which often contribute to chronic related diseases in the global population [3] - [5]. Thus, that makes every state infrastructure in the health department continues to campaign about the importance of diet and food behavior [6], [7]. Because it is known that the main factors that cause adverse effects on a person's weight status are physical inactivity and unhealthy dietary habits [5], [8]. Although very good knowledge of general guidelines for healthy eating, the occurrence of differences in the actions carried out by each person is strongly influenced by the perception factors that are built up in their minds [9].

Self-assessment is a well-established instrument in classifying someone who adopts a healthy lifestyle (preventive) or someone who does not maintain their lifestyle (curative). However self-assessment is known to have flaws because the results contain a bias which causes the accuracy of the classification results is not optimal. Many factors influence the misclassification done by selfassessment because everyone has their standardization in assessing themselves [10] - [12]. Then because the profile of eating habits is strongly influenced by one's views and perceptions, other alternatives using data obtained directly from their brains are believed to be able to support selfassessment to improve accuracy. Event-related potential (ERP) is the primary technique used by neuroscientists in dealing with identifying a person's perception or attention [13]. Therefore, the experiment in this study was conducted to capture and evaluate the performance of ERP brain data in recognizing their profile using machine learning techniques. This paper is organized as the following: 1) Introduction describes the background, problem statements, and research objectives of this study, 2) Related Works presents previous studies on ERP related to food, 3) Participants in this experiment are described, 4) Methodology demonstrates procedures or techniques used, 5) Discussion interprets and describes the findings of the experiment results, and 6) Conclusions and Future Work summarizes the main findings and suggest the recommendation to extend this current works.

\section{RELATED WORKS}

Event-related potential (ERP) is one technique for processing cognitive food images at the nerve level [14]. Research in using Event-related potential (ERP) has analyzed the reactivity of food images extensively [15]. ERP has revealed differences in the way the brain processes food images [16]. For research purposes, food images are a suitable and convenient alternative to real food because many dietary decisions are carried out based on the visual appearance of food [17]. 
In the past few years, there have been many experiments in utilizing ERP signals to understand a person's response to a stimulus in the form of a food image. Literature studies on cognitive ERP related to food is summarized in a study [18] which aims to look at ERP components that need to be highlighted concerning eating habits. This study concludes that ERP shows a convincing ability to extract features related to human cognition. However, there are limitations in several previous studies that only compare perceptions and attention to healthy and unhealthy food stimuli, or high calorie and low-calorie foods, but do not observe the relationship to eating habits. This raises the importance of holding this study to conduct further analysis with the relationship between ERP on food and eating habits [19-21].

As stated by previous studies, it was found that there is one powerful ERP cognitive component in showing differences in individual responses to attention processing in visual food images, namely the $\mathrm{P} 300$ ( $\left.\mathrm{P}_{3}\right)$ component [16], [18], [22]. P300 as the main component of research is a positive ERP component that occurs around $300 \mathrm{~ms}$ after the stimulus is given. This component is associated with the allocation of attention and memory in the human brain, where greater attention is reflected by larger $\mathrm{P} 3$ waves [23].

\section{PARTICIPANT}

Twenty-two (11 male, 11 females, mean age 21.45 years) undergraduate students at International Islamic University Malaysia (IIUM) participated in the experiment. Participants were prior recruited through public announcements and screened based on their scores and status on eating habits. This assessment was carried out using the HPLP-II questionnaire by taking the nutritional dimension. This kind of screening stage aims to find 11 participants with healthy eating habits status and 11 participants with unhealthy eating habit status to avoid having a dominant sample in this experiment. None of them reported any neurological disorders, eating disorders, substance abuse or addiction. All participants are explained about the experiment instructions and must be willing to sign the consent form before participation. This study was approved by the IIUM Research Ethics Committee (IREC).

\section{Methodology}

In general, the method or sequence of processes applied in this study is illustrated in Figure 1, namely data collection, pre-processing, and classification.

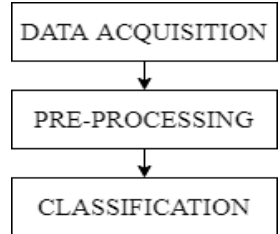

Fig. 1 The flow of methods used in this study

\section{A. Data Acquisition}

Raw EEG data was recorded using a Dabo-machine with 19 electrodes and EEG sampled at $250 \mathrm{~Hz}$. The location channel that was according to International 10-20 system includes Fp1, Fp2, F7, F3, Fz, F4, F8, T3, C3, Cz, C4, T4, T4, T5, P3, Pz, $\mathrm{P} 4, \mathrm{~T} 6, \mathrm{O}_{1}$, and $\mathrm{O}_{2}$. The electrode placement on the subject's scalp according to the position of the channel as shown in Figure 2, followed by an explanation of the electrode labelling in Table I. CFz indicates as a recording reference, and $A F z$ indicates as ground.

Prior the data collection session, there was a prerequisite for participants not to consume anything other than mineral water $(250 \mathrm{ml})$ within 3 hours beforehand [20]. This is because the hunger level becomes a new variable that affects data analysis.

Then participants were asked to sit comfortably while looking at the computer monitor used for stimulation presentations. During the recording of EEG data, participants passively pay attention to the images presented and were asked to minimize body movements. Participants' movements are monitored by researchers and documented via video for offline analysis.

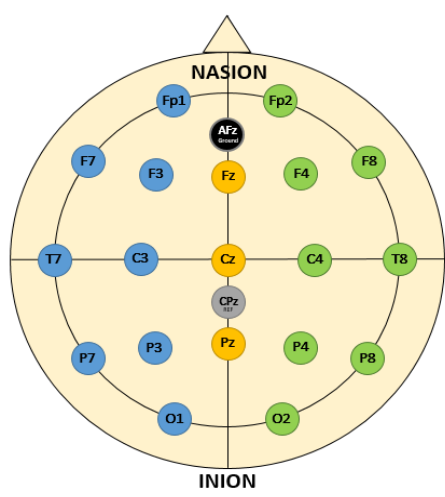

Fig. 2 Distribution of electrode sites on the scalp

TABLE I

EEG ELECTRODE LABELLING

\begin{tabular}{|c|c|c|c|}
\hline $\begin{array}{c}\text { Anatomical } \\
\text { Regions }\end{array}$ & Description & Left & Right \\
\hline Fp & Pre-frontal lobe & Fp1 & Fp2 \\
\hline F & Frontal lobe & F3, F7 & F4, F8 \\
\hline $\mathrm{T}$ & Temporal lobe & T7 & T8 \\
\hline $\mathrm{C}$ & Central & C3 & C4 \\
\hline $\mathrm{P}$ & Parietal lobe & P3, P7 & P4, P8 \\
\hline $\mathrm{O}$ & Occipital lobe & O1 & O2 \\
\hline $\mathrm{Z}$ & $\begin{array}{c}\text { An electrode placed } \\
\text { on the midline }\end{array}$ & - & - \\
\hline Nasion & $\begin{array}{c}\text { A point between the } \\
\text { forehead and nose } \\
\text { The bump at the } \\
\text { back of the skull }\end{array}$ & - & - \\
\hline Inion & $\begin{array}{c}\text { Refer to the right } \\
\text { hemisphere }\end{array}$ & - & - \\
\hline $\begin{array}{c}\text { Even } \\
\text { numbers }\end{array}$ & \multicolumn{2}{|c}{} \\
\hline
\end{tabular}


All participants were tested individually by receiving stimuli that were designed using the PsychoPy software. Each experiment begins with a background of light grey with a cross fixation in the middle with a duration of 1-2 seconds as an intertrial interval (ITI), followed by a visual presentation of stimulation in the form of food images for 2 seconds.

Stimuli consists of two categories of images that show images for healthy foods and unhealthy foods that are demonstrated randomly with ITI in between. A total of 60 stimuli, consisting of 30 images each for healthy and unhealthy food. The image database used in this study was taken from the Standardized Food Images (SFI) database [17]. Then the selection and categorization of images carried out by a nutritionist namely Dr Nur Fardian, M. Nutrition (Indonesia) and Asst. Prof. Dr Nor Azwani Binti Mohd Shukri (IIUM Kuantan, Malaysia).

\section{B. Pre-processing}

ERP data analysis is initialized by conducting preprocessing as shown in Figure 3. For the whole of operations in this method use a MATLAB package named ERPLAB Toolbox. This stage aims to clear data from noise or external sources not related to recorded neurological activity.

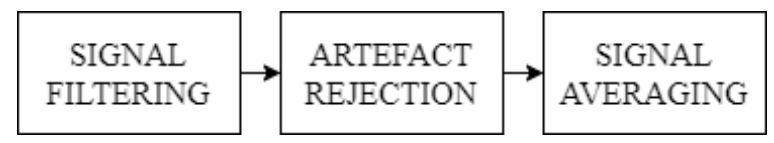

Fig. 3 The procedure flow of signal pre-processing

After receiving the raw EEG signals from each dataset, pre-processing begins with filtering of EEG signal recordings to eliminate signals from unwanted frequencies by pressing certain frequency areas without distorting and losing significant information from the data. This study uses a Bandpass filter by implementing a high-pass filter with a cutoff of half-amplitude $0.1 \mathrm{~Hz}$ and low-pass with a cut-off of half-amplitude of $30 \mathrm{~Hz}$.

Then proceed with the artefact rejection by Independent Component Analysis (ICA) which can extract some artifacts, especially ocular artifacts (blinking and eye movements) on EEG and ERP data. ICA is competent to increase SNR and maintain the integrity of information on brain activity [24] [27]

Subsequently since the noise has been minimized, the next step is to convert continuous EEG data into ERP brain signals for each subject. But to calculate the average of EEG signals, the process of segmentation and baseline correction needs to be done beforehand. Segmentation is performed by suppressing latency or time duration between 250-500 ms post-stimulus to extract the P300 component. Then ERPs are acquired by extracting and calculate the average of the EEG signals across a number of the same events [28] as in the following equation:

$$
y=\mathbb{E}\left[x_{i}\right] \approx \frac{1}{N} \sum_{j}^{N} x_{i}^{(j)}
$$

The variable $x_{i}$ refers to the raw EEG signal that reflects the activity associated with a specific period in the stimulation presentation for each electrode on the scalp. The parameters $N$ and $j$ refer to the number of trials and iteration index, respectively. Then the grand-averages are calculated for each group (i.e. preventive and curative) because ERP single subject analysis found that it is difficult and complex to capture the differences between groups and conditions.

\section{Classification}

The final step is the classification of eating habits profiles by using ERP data and dynamic evolving Spiking Neural Network (deSNN) technique. DeSNN is one of the artificial intelligence methods that is appropriate for studying several complexes from spatio-temporal brain data (STBD), such as ERP brain signals [29] - [33].

DeSNN has the advantage of adopting a rank-order mechanism so that it highlights the importance of sequences in the arrival of surges in input making it suitable for online learning in various applications [34]. DeSNN is also able to adjust connection weights depending on the next surge that comes at the same synapse over time so that the optimal ability is to capture a portion of memory (long term) through the potential growth of post-collapse [35].

This study uses Neucube software which is a machine that applies deSNN as a learning method. The NeuCube framework has been successfully applied to the classification and extraction of EEG data knowledge in numerous cases with high accuracy [30], [35] - [37].

\section{DISCUSSION}

\section{A. P300-ERP Results}

As a method for the initial analysis of the data on the P300 component, a visual inspection is performed by displaying a scalp topography using ERPLAB. Scalp topography shows temporal characteristics as oscillations or rhythms which extend over some time. Thus, for this frequency analysis method, Figure 4 shows a scalp topographic map over a range of $250-500 \mathrm{~ms}$ with $50 \mathrm{~ms}$ intervals for both preventive and curative profiles in healthy and unhealthy foods stimuli. Limiting the time range from 250 to $500 \mathrm{~ms}$ is carried out to analyze the P300 component that can be found in that time frame. Figure 4.a shows the scalp topography in the preventive group when receiving healthy food stimuli (top) and unhealthy food stimuli (bottom).

The topographic map is represented by a spectrum of colors with the legend on the right side of the image expressed in the scale bar for each group of profiles. The 
color spectrum shows the value of the voltage produced in each area or part of the scalp. Therefore, it can be seen that the darkest red shows the highest amplitude value, while the darkest blue shows the lowest amplitude value.
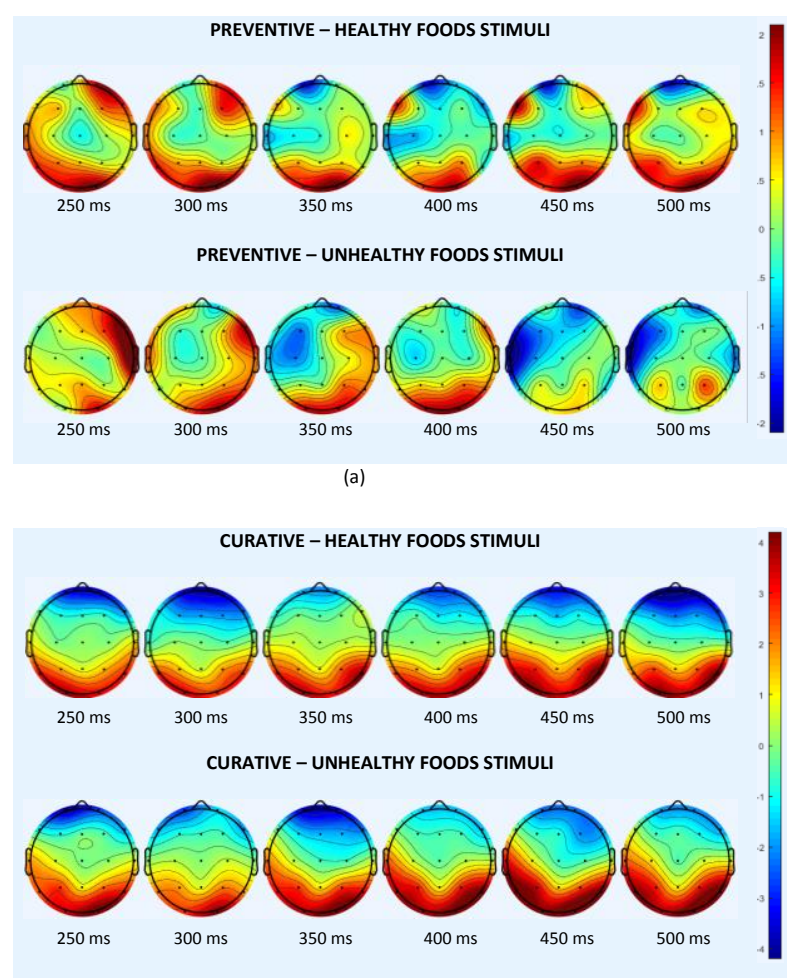

(b)

Fig. 4 Scalp topography on P300 component (250 - $500 \mathrm{ms)}$

Based on the examination of figure 4 , in both the preventive and curative groups it was found that in the posterior region located behind the head around the occipital and parietal electrodes $\mathrm{O}_{1}, \mathrm{O}_{2}, \mathrm{P}_{3}, \mathrm{P}_{4}, \mathrm{P}_{5}$ and $\mathrm{P} 7$ produced red color. This indicates that the voltage generated in the area is higher than in other areas. This result is in line with several previous studies which also discovered that the parietal and occipital regions are strong references in identifying perception and attention [32], [38], [39]. Therefore, further discussion is focused on the relevant lobes in the posterior region, namely the parietal and occipital lobes.

Furthermore, through visual exploration in Figure 4.a, an interesting initial interpretation can be taken that the preventive group produces a relatively greater amplitude for healthy food compared to unhealthy food stimulation. In contrast to the curative group in Fig 4.b, a greater amplitude is produced by unhealthy foods compared to healthy foods. The amplitude value is known to be larger because the area displays a wider red color. The red color is known to represent higher amplitude values. Therefore, with the initial analysis of the scalp topography, it was found that in the preventive profile, the attention generated by the subject was higher for healthy food. While on the curative profile, the attention was higher when the subjects received images of unhealthy food.

Also, the amplitude in the posterior region seems to increase intensively over time. For example, in the preventive group of healthy food stimuli (refer to Fig. 4.a), the red color appears darker and wider when observed from each scalp map capture at 200, 250, 300, 350, 400, 450, and 500. Similarly, the curative group on unhealthy foods (refer to Fig. 4.b), red is also darker and wider when it reaches greater latency.

The initial interpretation is further proven by calculating the average amplitude at 250-500 ms for each preventive and curative group. The mean amplitude results in channels $\mathrm{P}_{3}, \mathrm{P}_{4}, \mathrm{P} 7, \mathrm{P} 8, \mathrm{O} 1$, and $\mathrm{O}_{2}$ in all conditions are shown in table II and table III for preventive and curative group respectively.

TABLE III

Mean Amplitude of Preventive Group - P3Oo in PARIETAL ANd OCCIPITAL Lobe

\begin{tabular}{|c|c|c|}
\hline \multirow{2}{*}{ Channels } & \multicolumn{2}{|c|}{ Stimuli } \\
\cline { 2 - 3 } & Healthy Food & Unhealthy Food \\
\hline P3 & 1.05 & 0.56 \\
\hline P4 & 0.79 & 0.93 \\
\hline P7 & 0.91 & 0.17 \\
\hline P8 & 0.58 & 0.58 \\
\hline O1 & 1.61 & 1.14 \\
\hline O2 & 2.49 & 1.53 \\
\hline Mean & $\mathbf{1 . 2 4}$ & $\mathbf{0 . 8 2}$ \\
\hline
\end{tabular}

TABLE IIIII

Mean Amplitude of Curative Group - P3Oo in Parietal and Occipital Lobe

\begin{tabular}{|c|c|c|}
\hline \multirow{2}{*}{ Channels } & \multicolumn{2}{|c|}{ Stimuli } \\
\cline { 2 - 3 } & Healthy Food & Unhealthy Food \\
\hline P3 & 1.96 & 2.83 \\
\hline P4 & 2.23 & 2.52 \\
\hline P7 & 2.78 & 3.04 \\
\hline P8 & 3.81 & 3.48 \\
\hline O1 & 3.76 & 3.48 \\
\hline O2 & 3.35 & 4.94 \\
\hline Mean & $\mathbf{2 . 9 8}$ & $\mathbf{3 . 3 8}$ \\
\hline
\end{tabular}

Table II confirms that the mean amplitude produced in the preventive group in the parietal and occipital areas is greater for healthy food images. Then table III shows that the results for the curative group are contradictory compared to the preventive group where a greater mean amplitude is generated for unhealthy foods. The mean amplitude results are aligned and reinforce the initial interpretation of the scalp topography that has been reviewed previously.

The results of ERP signal analysis seem to show and reflect more desire and attention to the food image that is 
their preference in choosing food in their daily life. Based on a few papers that stated the amplitude seems greater when the subject allocates the more attention to the affective image [23], [40] - [42], then it can be interpreted that each group shows interest based on their food choices in everyday life. The preventive group that maintained their health showed a greater interest in healthy foods. Meanwhile, the curative group who tended not to take concern to their dietary nutrition showed more attention to unhealthy foods.

\section{B. DeSNN Results}

The following are the results of the $\mathrm{P}_{3}$ wave classification process using the dynamic evolving spiking neural network technique. The dataset is designed with some information shown in Table IV. The 19 features refer to the number of channels used, the length of time is the number of recorded data as many as 63 representing ERP data that is locked time for 252-500 ms, and 2 classes show as a label of the classification process, namely preventive (class 1) and curative (class 2).

TABLE IVV

DATASET INFORMATION ON P3OO-ERP CLASSIFICATION

\begin{tabular}{|c|c|}
\hline Dataset Component & Number \\
\hline Sample & 22 \\
\hline Feature & 19 \\
\hline Time Length & 63 \\
\hline Class & 2 \\
\hline
\end{tabular}

Further, the classification is done through a 0.5 set training ratio process and verifies the classification with leave-oneout cross validation. Table $V$ records the accuracy of ERP data on two stimuli, namely healthy food and unhealthy food. This demonstrates that the proposed method is proven to run in this study.

Referring to Table $\mathrm{V}$, the average accuracy of the test results on the waveform of healthy foods is the same as unhealthy foods. The resulting accuracy tends to be low. This is most likely due to the relatively small number of samples to be able to build a good profiling model. As it is known that Neucube will show better performance if given greater training data [32], [34], [43], [44].

TABLE V

DESNN RESULT ON P300-ERP DATA

\begin{tabular}{|c|c|c|c|}
\hline Accuracy & $\begin{array}{c}\text { Healthy } \\
\text { Food }\end{array}$ & $\begin{array}{c}\text { Unhealthy } \\
\text { Food }\end{array}$ & $\begin{array}{c}\text { Difference } \\
\text { Wave }\end{array}$ \\
\hline Class 1 & $18.18 \%$ & $36.36 \%$ & $27.27 \%$ \\
\hline Class 2 & $63.64 \%$ & $45.45 \%$ & $27.27 \%$ \\
\hline Average & $\mathbf{4 0 . 9 1 \%}$ & $\mathbf{4 0 . 9 1 \%}$ & $\mathbf{2 7 . 2 7 \%}$ \\
\hline
\end{tabular}

Furthermore, based on Table V, it can be seen that both in healthy or unhealthy food stimuli, more classification difficulties occur when predicting preventive class. It also can be seen that in class 1 , which represents the preventive group, the accuracy is very low compared to the curative group or class 2. This means that the deSNN technique has difficulty in identifying and classifying the preventive class. This shows that the chosen subject seems unable to represent the profile of healthy eating to the best possible way. So, in addition to adding sample data, the quality of the subject also needs to be taken into account to be improved as well. This can be done by recruiting participants by determining target subjects in the form of sportsmen or nutritionists who are considered to have a high awareness of the importance of a good lifestyle.

\section{CONCLUSIONS AND FUTURE WORK}

Preventive or healthy eating habits group generated the greater amplitude generated in the healthy food stimuli, while the curative or unhealthy eating habits group generated a greater amplitude in the unhealthy food. These results support the belief that food images are closely related to food choices in everyday life. It was found that the ERP component of the $\mathrm{P} 300$ shows promising potential for further analysis in identifying a person's profile based on their eating habit. The proposed methods using ERP data and dynamic evolving spiking neural networks in eating habit profiling are proven to be operational. The accuracy rate of deSNN might be higher if the number of samples is enlarged and the quality of the subject is improved.

In future work, the number of subjects and the quality of qualifications must be increased to present each group's profile more clearly. To improve the quality of the subject, it can be conducted by setting targets by specifying an environment for distributing questionnaires, for example, sports areas or student's degree in the health field for finding the extremely preventive people.

The experiment protocol used is also recommended to be more varied by assigning instructions on viewing unhealthy food stimuli, such as direction for thinking about the taste of the food, imagining eating the food or thinking about the short- and long-term consequences of eating the food. It is expected that by applying all the suggestions and recommendations presented, classification accuracy can be improved and a profiling method can be built properly.

\section{ACKNOWLEDGMENT}

This research was supported by the Fundamental Research Grant Scheme FRGS/1/2016/ICT02/UIAM/02/2 I FRGS16-029-0528 from the Ministry of Higher Education (MOHE) Malaysia. We are grateful to all the members at Research Unit 9: Computational Intelligence Research Group (CIRG) and Research Unit 2: Pervasive Computing for Brain Development Research Group (PCBDG), Kulliyyah of 
Information and Communication Technology (KICT), IIUM, for helpful advice and kind discussion.

\section{REFERENCES}

[1] Abraham, S., B. R. Noriega, and J. Y. Shin. "College students eating habits and knowledge of nutritional requirements," Journal of and Human Health, vol. 2, no. 1 (2018), pp. 13-17.

[2] Brown, Onikia N., Lauren E. O'Connor, and Dennis Savaiano. "A pilot study using text messaging to provide nutrition education and promote better dietary choices in college students," Journal of American College Health, vol. 62, no. 5, 2014, 320-327.

[3] World Health Organization. Obesity and overweight. http://www.who.int/mediacentre/factsheets/fs311/en/. ～Updated 2020. Accessed July 25, 2020.

[4] Twells, Laurie K., Deborah M. Gregory, Jacinta Reddigan, and William K. Midodzi. "Current and predicted prevalence of obesity in Canada: a trend analysis," CMAJ open 2, no. 1, 2014, E18-E26.

[5] Allom, Vanessa, and Barbara Mullan. "Maintaining healthy eating behaviour: experiences and perceptions of young adults," Nutrition \& Food Science, Vol. 44 No. 2, 2014, pp. 156-167. Swinburn, Boyd A., Gary Sacks, Kevin D. Hall, Klim McPherson, Diane T. Finegood,

[6] Marjory L. Moodie, and Steven L. Gortmaker. "The global obesity pandemic: shaped by global drivers and local environments," The Lancet, vol. 378, no. 9793, 2011, pp. 804-814. Monteiro, Carlos A., J c.

[7] Moubarac, Geoffrey Cannon, Shu Wen Ng, and Barry Popkin. "Ultra - processed products are becoming dominant in the global food system," Obesity reviews, vol. 14, 2013, pp. 21-28.

[8] Hutchesson, Melinda J., Megan E. Rollo, Rebecca Krukowski, Louisa Ells, Jean Harvey, Philip J. Morgan, Robin Callister, Ronald Plotnikoff, and Clare E. Collins. "eHealth Interventions for The Prevention and Treatment of Overweight and Obesity in Adults: A Aystematic Review with Meta - analysis." Obesity reviews, vol. 16, no. 5, 2015, pp. 376-392.

[9] Sherry, Diana S. "Does Knowledge of Evolutionary Biology Change High School Students' Attitudes About Healthy Eating?," Evolution: Education and Outreach, vol. 12, no. 1, 2019, pp. 19-29.

[10] Crandall, Virginia C., Vaughn J. Crandall, and Walter Katkovsky. "A children's social desirability questionnaire," Journal of consulting psychology 29, no. 1, 1965, pp. 27-36.

[11] Van de Mortel, Thea F. "Faking it: social desirability response bias in self-report research," Australian Journal of Advanced Nursing, vol. 25, no. 4, 2008, pp. 40-48.

[12] Rosenman, Robert, Vidhura Tennekoon, and Laura G. Hill. "Measuring bias in self-reported data," International Journal of Behavioural and Healthcare Research, vol. 2, no. 4, 2011, pp. 320-332.

[13] Näätänen, Risto, and Patricia T. Michie. "Early selective-attention effects on the evoked potential: a critical review and reinterpretation." Biological psychology, vol. 8, no. 2, 1979, pp. 81-136.

[14] Carbine, Kaylie A., Kara M. Duraccio, C. Brock Kirwan, Nathan M. Muncy, James D. LeCheminant, and Michael J. Larson. "A direct comparison between ERP and fMRI measurements of food-related inhibitory control: Implications for BMI status and dietary intake," Neurolmage, vol. 166, 2018, pp. 335-348.

[15] Schwab, Daniela, Matteo Giraldo, Benjamin Spiegl, and Anne Schienle. "Disgust evoked by strong wormwood bitterness influences the processing of visual food cues in women: An ERP study," Appetite, vol. 108, 2017, pp. 51-56.

[16] Feig, Emily H., Samantha R. Winter, John Kounios, Brian Erickson, Staci A. Berkowitz, and Michael R. Lowe. "The role of hunger state and dieting history in neural response to food cues: An event-related potential study," Physiology \& behavior, vol. 179, 2017, pp. 126-134

[17] Charbonnier, Lisette, Floor van Meer, Laura N. van der Laan, Max A. Viergever, and Paul AM Smeets. "Standardized food images: a photographing protocol and image database," Appetite, vol. 96, 2016, pp. 166-173.
[18] Carbine, Kaylie A., Rebekah Rodeback, Erin Modersitzki, Marshall Miner, James D. LeCheminant, and Michael J. Larson. "The utility of event-related potentials (ERPs) in understanding food-related cognition: A systematic review and recommendations." Appetite, vol. 128, 2018, pp. 58-78.

[19] Carbine, K. A., Christensen, E., LeCheminant, J. D., Bailey, B. W., Tucker, L. A., and Larson, M. J. "Testing Food-Related Inhibitory Control to High- and Low-Calorie Food Stimuli Electrophysiological Responses to High-Calorie Food Stimuli Predict Caloric Intake," Psychophysiology, vol. 54, 2017, pp. 982-997.

[20] Kong, Fanchang, Yan Zhang, and Hong Chen. "Inhibition ability of food cues between successful and unsuccessful restrained eaters: a two-choice oddball task," PLoS One, vol. 10, no. 4, 2015, pp. 1-12.

[21] Meule, A., Kubler, A., \& Blechert, J. "Time course of electrocortical food-cue responses 856 during cognitive regulation of craving," Frontiers in Psychology, vol. 4, 2013, pp. 1-11.

[22] Becker, Christoph A., Tobias Flaisch, Britta Renner, and Harald T. Schupp. "Neural correlates of the perception of spoiled food stimuli," Frontiers in human neuroscience, vol. 10, 2016, pp. 302-312.

[23] Wu, Jia, Cynthia J. Willner, Claire Hill, Pasco Fearon, Linda C. Mayes, and Michael J. Crowley. "Emotional eating and instructed food-cue processing in adolescents: An ERP study," Biological psychology, vol. 132, 2018, pp. 27-36.

[24] Bobe, Anatoly S., Andrey S. Alekseev, Maria V. Komarova, and Dmitry Fastovets. "Single-trial ERP Feature Extraction and Classification for Visual Object Recognition Task," In 2018 Engineering and Telecommunication (EnT-MIPT), IEEE, 2018, pp. 188-192.

[25] Pontifex, Matthew B., Kathryn L. Gwizdala, Andrew C. Parks, Martin Billinger, and Clemens Brunner. "Variability of ICA decomposition may impact EEG signals when used to remove eyeblink artifacts," Psychophysiology vol. 54, no. 3, 2017, pp. 386-398.

[26] Dharmaprani, Dhani, Hoang K. Nguyen, Trent W. Lewis, Dylan DeLosAngeles, John O. Willoughby, and Kenneth J. Pope. "A comparison of independent component analysis algorithms and measures to discriminate between EEG and artifact components," In 2016 38th Annual International Conference of the IEEE Engineering in Medicine and Biology Society (EMBC), IEEE, 2016, pp. 825-828.

[27] Plöchl, Michael, José Pablo Ossandón, and Peter König. "Combining EEG and eye tracking: identification, characterization, and correction of eye movement artifacts in electroencephalographic data." Frontiers in human neuroscience, vol. 6, 2012, pp. 278-300.

[28] Luck, Steven J., Geoffrey F. Woodman, and Edward K. Vogel. "Eventrelated potential studies of attention," Trends in cognitive sciences, vol. 4, no. 11, 2000, pp. 432-440.

[29] Kasabov, Nikola. "Evolving spiking neural networks and neurogenetic systems for spatio-and spectro-temporal data modelling and pattern recognition," In IEEE World Congress on Computational Intelligence, Springer, Berlin, Heidelberg, 2012, pp. 234-260.

[30] Doborjeh, Maryam Gholami, and Nikola Kasabov. "Personalised modelling on integrated clinical and EEG spatio-temporal brain data in the NeuCube spiking neural network system," In 2016 International Joint Conference on Neural Networks (IJCNN), IEEE, 2016, pp. 13731378.

[31] Doborjeh, Zohreh G., Maryam Doborjeh, and Nikola Kasabov. "EEC Pattern Recognition using Brain-Inspired Spiking Neural Networks for Modelling Human Decision Processes," In 2018 International Joint Conference on Neural Networks (IJCNN), IEEE, 2018, pp. 1-7.

[32] Doborjeh, Zohreh Gholami, Nikola Kasabov, Maryam Gholam Doborjeh, and Alexander Sumich. "Modelling peri-perceptual brain processes in a deep learning spiking neural network architecture," Scientific reports, vol. 8, no. 1, 2018, pp. 1-13.

[33] Capecci, Elisa, Nikola Kasabov, and Grace Y. Wang. "Analysis of connectivity in NeuCube spiking neural network models trained on EEG data for the understanding of functional changes in the brain: $A$ case study on opiate dependence treatment," Neural Networks, vol. 68, 2015, pp. 62-77. 
[34] Kasabov, Nikola, Nathan Matthew Scott, Enmei Tu, Stefan Marks, Neelava Sengupta, Elisa Capecci, Muhaini Othman et al. "Evolving spatio-temporal data machines based on the NeuCube neuromorphic framework: design methodology and selected applications," Neural Networks, vol. 78, 2016, pp. 1-14.

[35] Kasabov, Nikola, Kshitij Dhoble, Nuttapod Nuntalid, and Giacomo Indiveri. "Dynamic evolving spiking neural networks for on-line spatio-and spectro-temporal pattern recognition," Neural Networks, vol. 41, 2013, pp. 188-201.

[36] Hu, Jin, Zeng-Guang Hou, Yi-Xiong Chen, Nikola Kasabov, and Nathan Scott. "EEG-based classification of upper-limb ADL using SNN for active robotic rehabilitation," In 5th IEEE RAS/EMBS international conference on biomedical robotics and biomechatronics, IEEE, 2014, pp. 409-414.

[37] Capecci, Elisa, Francesco Carlo Morabito, Maurizio Campolo, Nadia Mammone, Domenico Labate, and Nikola Kasabov. "A feasibility study of using the neucube spiking neural network architecture for modelling alzheimer's disease eeg data," In Advances in neural networks: Computational and theoretical issues, Springer, Cham, 2015, pp. 159-172.

[38] Lu, Zhaohua, Qi Li, Ning Gao, Jingjing Yang, and Ou Bai. "Happy emotion cognition of bimodal audiovisual stimuli optimizes the performance of the P300 speller," Brain and Behavior, vol. 9, no. 12, 2019, pp. 1-13
[39] Hamish Innes-Brown, Ayla Barutchu, and David P. Crewther. "Neura Responses in Parietal and Occipital Areas in Response to Visual Events Are Modulated by Prior Multisensory Stimuli," PLOS ONE, vol. 8, no. 12, pp. 1-13.

[40] Klasen, M., Kreifelts, B., Chen, Y. H., Seubert, J., and Mathiak, K. "Neural Processing of Emotion in Multimodal Settings," Frontiers in human neuroscience, vol. 8, 2014, pp. 822-825.

[41] Sur, S., \& Sinha, V. K. "Event-related potential: An overview," Industrial psychiatry journal, vol. 18, no. 1, 2009, pp. 70-73.

[42] Woodman, G. F. "A Brief Introduction to The Use of Event-Related Potentials in Studies of Perception and Attention," Attention, Perception, \& Psychophysics, vol. 71, no. 6, 2010, pp. 1233-1240.

[43] Kasabov, Nikola K., Maryam Gholami Doborjeh, and Zohreh Gholami Doborjeh. "Mapping, learning, visualization, classification, and understanding of $\mathrm{FMRI}$ data in the NeuCube evolving spatiotemporal data machine of spiking neural networks," IEEE transactions on neural networks and learning systems, vol. 28, no. 4, 2016, pp. 887899.

[44] Tu, Enmei, Nikola Kasabov, and Jie Yang. "Mapping temporal variables into the neucube for improved pattern recognition, predictive modeling, and understanding of stream data." IEEE transactions on neural networks and learning systems, vol. 28, no. 6, 2016, pp. 1305-1317 